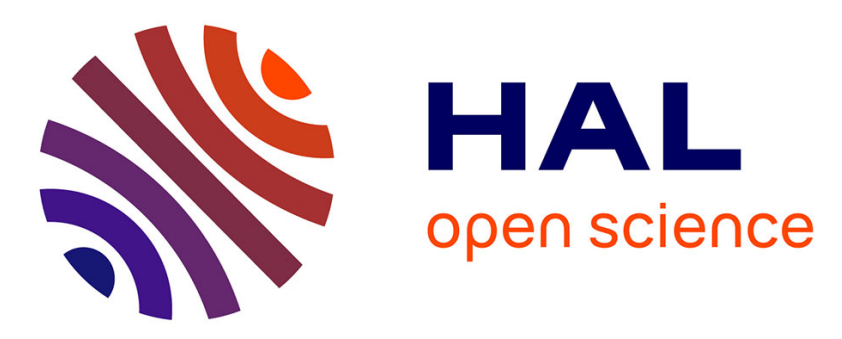

\title{
The role of the dorsal anterior insula in ecstatic sensation revealed by direct electrical brain stimulation
}

Fabrice Bartolomei, Stanislas Lagarde, D. Scavarda, R. Carron, C.G. Bénar, F. Picard

\section{- To cite this version:}

Fabrice Bartolomei, Stanislas Lagarde, D. Scavarda, R. Carron, C.G. Bénar, et al.. The role of the dorsal anterior insula in ecstatic sensation revealed by direct electrical brain stimulation. Brain Stimulation, 2019, 12 (5), pp.1121-1126. 10.1016/j.brs.2019.06.005 . hal-02513939

\author{
HAL Id: hal-02513939 \\ https://hal.science/hal-02513939
}

Submitted on 20 Dec 2021

HAL is a multi-disciplinary open access archive for the deposit and dissemination of scientific research documents, whether they are published or not. The documents may come from teaching and research institutions in France or abroad, or from public or private research centers.
L'archive ouverte pluridisciplinaire HAL, est destinée au dépôt et à la diffusion de documents scientifiques de niveau recherche, publiés ou non, émanant des établissements d'enseignement et de recherche français ou étrangers, des laboratoires publics ou privés.

\section{(ㄷ)(1) $\$$}

Distributed under a Creative Commons Attribution - NonCommerciall 4.0 International 
The role of the dorsal anterior insula in ecstatic sensation revealed by direct electrical brain stimulation

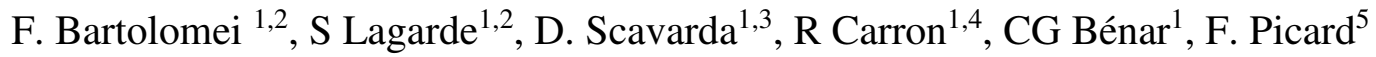

1. Aix Marseille Univ, INSERM, INS, Inst Neurosci Syst, Marseille, France

2. APHM, Timone Hospital, Clinical Neurophysiology and Epileptology Department, Marseille, 13005, France

3. Department of Pediatric Neurosurgery, Timone University Hospital, Marseille, France.

4. Department of Functional and Stereotactic Neurosurgery, Timone University Hospital, Marseille, France.

5. Department of Neurology, University Hospitals and Medical School of Geneva, Geneva, Switzerland

\section{Corresponding Author:}

Prof Fabrice Bartolomei, MD, PhD

Address: Service d'Epileptologie et Rythmologie Cérébrale, CHU Timone - 264 Rue St Pierre 13005-Marseille, France

Tel: +33491385833 Fax: +33491385826 Email: Fabrice.bartolomei@ap-hm.fr 


\begin{abstract}
Background: An ecstatic phenomenon is an altered state of consciousness with a sense of "hyper-reality", and a complete present-moment awareness with a feeling of union with the Universe. A better understanding of the network mechanisms underlying this fascinating subjective experience may help to unravel some mysteries of human consciousness. Insula has been recently proposed to be a key region to elicit these symptoms.

Objective/Hypothesis: We studied functional connectivity changes in several brain areas during the induction of ecstatic auras by direct electrical stimulation of the dorsal anterior insular cortex in patients with refractory focal epilepsy implanted with intracerebral electrodes (stereotactic-EEG, SEEG) in the context of their pre-surgical evaluation.

Methods: Three patients were selected on the basis of the occurrence of ecstatic symptoms triggered by direct intracerebral electrical stimulation (ES) of the antero-dorsal part of the insula. ES was performed (50 Hz, 1.5-2.1 mA, in a bipolar fashion to each contact in the gray matter during a 3 second period) to map functional cortices and trigger habitual seizures. One stimulation inducing ecstatic changes in each patient was analyzed. Functional connectivity analysis was performed by measuring interdependencies (nonlinear regression analysis based on the $h^{2}$ coefficient) between SEEG signals before and after stimulations.

\section{Results:}

In all patients, only the stimulation of dorsal anterior insula was able to reproduce an ecstatic aura. We observed a significant increase of functional connectivity values between several brain regions in the immediate period following stimulations. The most commonly implicated region was the dorsal anterior insula. Out-degrees (a measure intended to identify leading structures in a network) identified the dorsal anterior insula as the most common leading region in the induced networks.
\end{abstract}

Conclusion(s): Our findings bring additional support in favor of a major role played by the dorsal anterior insula in ecstatic experiences.

\title{
Highlights
}

- In 3 patients, only the direct stimulation of the dorsal anterior insula induced ecstatic phenomenon. 
- Increased functional connectivity in the period post stimulation implicated mostly the anterior insula

- Outdegrees identified the dorsal anterior insula as the most common leading region.

\section{Introduction}

The epileptic "aura" is a subjective phenomenon that sometimes precedes the visible clinical features of a seizure and that is related to the beginning of seizures in the brain. A few patients with epilepsy experience a type of aura referred to as "ecstatic". Ecstatic epileptic auras consist of a sensation of "hyper-reality". The patients use terms such as "clarity", "evidence", "certainty", "understanding", "insight", "enlightenment" or "epiphany". Dostoevsky gave a famous description of such seizures, that he experienced himself and in which he reached a wonderful state of clarity and bliss [1]. The phenomenological detail of the ecstatic aura includes three important points: bliss ("the immense joy that feels me is above physical sensations"[2]), a sense of certainty ("things suddenly seemed self-evident"[3]), and a sense of timelessness or a sense of being in an eternal now ("These moments are without beginning and without end"[2]). The feeling of certainty during ecstatic auras and a frequent feeling of oneness with the Universe are sometimes interpreted as mystical or religious by some individuals, depending on their previous religious beliefs, and may change their life forever.

The suspected location of the ecstatic ictal symptoms was the temporal lobe until recently, however evidence has converged more recently pointing to an involvement of the dorsal anterior insula probably within a larger neural network [2, 4, 5]. We could support this hypothesis of insular involvement by the finding of a first patient in whom the direct stimulation of the antero-dorsal part of the insula induced her ecstatic auras [6]. In the present report, we studied functional connectivity changes in several brain areas during the induction of ecstatic auras by electrical stimulation of the dorsal anterior insula in 3 patients having intracerebral electrodes for presurgical evaluation. Physiological functions in the brain require the functional interactions between multiple distinct neural networks. The measure of statistical interdependencies between signals of brain activity is an estimation of functional interactions and is referred to as "functional connectivity"[7]. The underlying assumption is 
that such correlations reflect, at least in part, functional interactions between different brain areas.

\section{Materials and methods}

\section{Selection of patients}

Subjects were 3 patients (P1, P2 and P3) (Table 1) with drug-resistant focal epilepsy, candidate to surgical treatment. They were studied in the Epilepsy Unit of the Timone Hospital, Marseille, France. For all these patients, intracerebral recordings with stereotactic EEG (SEEG) were performed in the context of their pre-surgical evaluation. These three patients were selected in the period 2011-2017 because they reported during intracerebral stimulation the occurrence of ecstatic symptoms defined by seizures with the feeling of (1) intense positive emotion (bliss), (2) enhanced physical well-being, and (3) heightened selfawareness or heightened perception of the external world (clarity) [2]. These feelings were similar to those reported during their spontaneous auras. Patients with only vague pleasant phenomenon, with orgasmic/sexual feeling or with associated perceptual hallucinations (feeling of a presence, auditory hallucinations...) were not selected. Patients signed informed consent, and the study was approved by the Institutional Review board (IRB00003888) of INSERM. Patient data are summarized in Table 1.

\section{SEEG recordings and electrical stimulation}

SEEG recordings were made thanks to intracerebral electrodes with multiple contacts (10-15 contacts) as previously described [8]. The electrodes were positioned in each patient based upon hypotheses about the localization of the epileptogenic zone, formulated from available noninvasive information. Each patient had a comprehensive evaluation including detailed history, neurological examination, neuropsychological testing, high resolution magnetic resonance imaging (MRI), and video-electroencephalography (EEG) recording of seizures before SEEG. Signals were recorded on a 256-channels Natus ${ }^{\mathrm{TM}}$ system. They were sampled at $1024 \mathrm{~Hz}$. In total, 3 stimulations in 3 different patients, were selected for this study. Electric stimulation was produced by a regulated neurostimulator designed for safe diagnostic stimulation of the human brain (Inomed $\left.{ }^{\circledR}\right)$. High frequency stimulation at $50 \mathrm{~Hz}$ (pulse duration 1 millisecond, intensity 0.5-2 $\mathrm{mA}$ ) was applied in a bipolar fashion to each of the 
pairs of contacts in the gray matter during a 3-5 seconds period. During stimulation, patient was sitting in bed and was asked to read or count.

One stimulation inducing ecstatic changes in each patient was analyzed (table 1). In each patient we studied the interdependencies between bipolar SEEG signals (i.e., difference of contiguous contacts).

\section{SEEG analysis: estimation of functional connectivity changes}

Functional connectivity is defined as the statistical dependencies between two neurophysiological responses. In our case, these functional connectivity measures are directed, in the sense that we assessed nonlinear correlations as a function of time lag between electrodes.

\section{Definition of the period of interest}

For each stimulation we chose two periods of analysis. The background period was a period of $15 \mathrm{~s}$ immediately preceding the stimulation and is referred here as PreS ("pre stimulation"). The second period was a $10 \mathrm{~s}$ period immediately following the stimulation artifact and referred as PoS (post stimulation). All the analyses were performed with bipolar derivation (subtraction of consecutive channels). During stimulation, the bipolar contacts are disconnected and cannot be studied for connectivity analysis. Thus we have taken two adjacent contacts representative of the stimulated region.

Signals were filtered in Anywave software [9] (software available at http://meg.univamu.fr/wiki/AnyWave), with Butterworth filters of order 4 . We used only a $1 \mathrm{~Hz}$ high pass filter in all the analyses.

\section{Estimation of functional connectivity using Non-linear correlation $\left(h^{2}\right)$}

Signals were analyzed with the AnyWave software [9], which computes a nonlinear regression analysis based on the $\mathrm{h}^{2}$ coefficient[10] . In summary, a piecewise linear regression is performed between each pair of signals, testing all the shifts of one signal relative to the other within a maximum lag. The $\mathrm{h}^{2}$ is the coefficient of determination which measures the goodness of fit of the nonlinear regression - equivalent to the R-squared used in linear regression. The $\mathrm{h}^{2}$ is bounded between 0 (no correlation) and 1 (maximal correlation) and contrary to the R-squared - is not symmetric [11]. We used a sliding window of 3 seconds with an overlap of 2 seconds, and a maximum delay between signals of $100 \mathrm{~ms}$. For each pair of signals $(\mathrm{X}, \mathrm{Y})$, we retained the maximal value across delays and across directions $(\mathrm{X}$ to $\mathrm{Y}$ 
or $\mathrm{Y}$ to $\mathrm{X}$ ). This value corresponded to a delay between signals, which was used to define the direction of the link. We thus obtained functional connectivity graphs, with each channel representing a node of the graph, and $h^{2}$ values the strength of the link between two nodes[12].

Connectivity graphs were obtained for the three stimulations (one for each patient) between bipolar signals derived from the grey matter of 7 regions. We computed all pair-wise interactions from 7 regions (42 connections), and for each connectivity pair, we took the maximum (resulting in a final number of 21 interactions). For clinical reasons, the sampling of the brain could be variable from one patient to the other. Therefore we selected a common set of regions (amygdala, hippocampus, temporal neocortex, dorsal anterior insular cortex, orbitofrontal cortex, dorsolateral prefrontal cortex (DLPFC)) and another neocortical region that was variable from one patient to the other (see Fig 2 for the electrodes placement on 3D mesh of the MRI in the three patients).

\section{Graph measures}

We summarized the connectivity graphs with a graph measures, the node degrees. This entailed thresholding the graphs, and then counting the number of significant links between a given node and the rest of the graph. This has the advantage of removing the links with low connectivity. The threshold was set empirically to 0.2 . In order to study the influence of the choice of threshold on the results, we also estimated the degrees using thresholds at 0.1 and 0.3. Overall the results are very similar (see Figure in supplementary material) and in the following, only results at a threshold of 0.2 will be reported.

Thresholding resulted in binary graphs (a link is 0 or 1 ), with each node corresponding to a channel, and a direction given by the delay. In other words, we assembled an asymmetric and directed adjacency matrix, based upon our measures of directed functional connectivity. Then, in each time window and for each channel, we counted the number of links with an $\mathrm{h}^{2}$ value above the threshold, only for the outgoing links (OUT measure, corresponding to "out degrees" in graph theory), ingoing links (IN measure), and for all links independently of the directionality (TOT measure, with TOT=IN+OUT). High values of out-degrees OUT were considered to be indicative of the driving (i.e., source) regions [12].(see also supplementary material for more details)

\section{Graph comparison}


For each link, the $\mathrm{h}^{2}$ values were compared between PoS and PreS conditions. The test for a difference was performed across time windows (i.e., within subject) to compare two sets of functional connectivity measures for a given pairs of electrodes (one for PreS, one for PoS). We also compared the degrees for each given region. For both comparisons, we used a Wilcoxon non-parametric paired test with Bonferroni correction for multiple comparisons.

\section{Results}

The clinical responses obtained in the three patients are summarized in Table 1. The seizure onset zone spared the insula in the three patients, but the insula was involved during the seizure spread. Patient 1 has already been reported in a previous study[6]. Patients 2 and 3 also reported typical ecstatic sensations during their habitual seizures.

In all the three cases, the stimulated contacts were located in the antero-dorsal part of the insular cortex (two right side, one left side, Fig 1). The stimulations ranged from 1.6 to 2.1 $\mathrm{mA}(50 \mathrm{~Hz}, 1 \mathrm{~ms}$ pulse width, 3-5 sec) and were not followed by any after-discharge. The patients were asked what they felt immediately after the stimulation ended. In two subjects a directed interview focusing on the ecstatic phenomenon was performed. In all three cases, it was not possible to precisely detect the timing of occurrence within or after the stimulation period but the ecstatic feeling lasted a few seconds. In the three patients, the other stimulations (particularly in the mesial temporal lobe) failed to trigger such phenomenon.

Figure 2A shows the functional connectivity graphs superposed on a $3 \mathrm{D}$ rendering of the cortex, with each bipolar channel representing a node of the graph. Links represents the strengths of $h^{2}$ values between pairs of nodes with a significative change ( $p<0.001190$ for multiple comparison) between post stimulation ( $\mathrm{PoS})$ and pre stimulation (PreS) periods, during ecstatic symptoms induced by stimulation. These stimulations induced a significant increase of functional connectivity values between different regions, particularly between amygdala and hippocampus, dorsal anterior insula and prefrontal cortex, amygdala and orbitofrontal cortex, amygdala and dorsal anterior insula in P1; between dorsal anterior insula and orbitofrontal cortex, dorsal anterior insula and DLPFC, dorsal anterior insula and temporal neocortex, dorsal anterior insula and amygdala in P2; between dorsal anterior insula and amygdala, amygdala and DLPFC, amygdala and dorsolateral prefrontal cortex in P3. 
Figure 2B presents the differences in mean total degree (number of significant links, independently of the direction of the links) between PreS and PoS periods. The following regions increased significantly their degree values: P1: amygdala, hippocampus, dorsal anterior insula, orbitofrontal cortex, dorsolateral prefrontal cortex; P2: dorsal anterior insula; P3: amygdala, dorsal anterior insula, premotor cortex, dorsolateral prefrontal cortex.

Thus, variable changes in functional connectivity occurred after stimulation, but the most commonly implicated region in the three patients was the dorsal anterior insula. Out-degrees were estimated with a 0.2 threshold, in the PoS period. This measure is a mean of identifying leading structures in a network. The Fig 3A shows the out-degree values (colored spheres). For P1, two regions presented the highest values, the dorsal anterior insula and the frontal lobe. In P2 and P3 the highest value was observed in the insular cortex. The out-degree values pooled from the three stimulations are shown in the Fig 3B showing that the insula was the structure with the largest changes in the three patients.

\section{Discussion}

We report here the induction of ecstatic auras by electrical stimulation of the dorsal anterior insula in 3 patients with refractory focal epilepsy. To our knowledge, these are the only descriptions of true ecstatic auras induced by brain electrical stimulation in the literature. In our 3 patients, no other stimulated areas than the dorsal anterior insula could induce such symptoms. We demonstrated that, at the time of the induction of the ecstatic symptoms, the insula was the most active region, playing a leading role among different areas forming an hyperactive network. Importantly, the analysis was performed in the few seconds following the stimulation, thus avoiding the bias related to the stimulation artifact.

The anterior insula has been recognized as a major component of the salience network, in association with the dorsal anterior cingulate cortex. The anterior insula activates in cases of behaviorally-relevant new external or internal stimuli $[13,14]$. The insula also integrates the interoceptive signals, i.e. stimuli coming from the inside of the body. It processes the physiological body state, contextualized by exteroceptive (environmental) signals. In the context of predictive coding in the brain, the insula continuously produces (top down) interoceptive predictions and generates prediction errors when the real signals arrive, allowing to update the next predictions. This process of interoception participates in the generation of emotions, feelings and probably the sense of self $[13,14]$. The neurocognitive hypothesis in ecstatic seizures is that an aberrant epileptic activity affecting a network which includes the 
anterior insula could prevent it from reaching the minimal level of differentiation and complexity necessary to encode predictions and/or prediction errors related to interoception and emotions. This would mimic a perfect match between interoceptive predictions and the incoming interoceptive signals, leading to a sense of certainty [3,5]. Such a state was described as the "ultimate stable state", which Mumford suggested occurred when the topdown cortical signals would perfectly predict representations at lower cortical levels[15]. This would be the state of a perfect prediction of the world that the brain is trying to achieve, in order to avoid surprise and minimize energy expenditure.

There are two circumstances in which this sense of certainty and consequent bliss as experienced in ecstatic auras occurs. First, the "Aha!" moment or "eureka" moment (also known as insight) is an experience of sudden understanding of a previously incomprehensible problem or concept, accompanied by joy or satisfaction. A recent study showed a specific activation of the dorsal anterior insula at an eureka moment of bug detection in computer code [16]. A previous study on insight solutions also showed an early bilateral insular activation [17]. Secondly, a peaceful and blissful state similar to the ecstatic state can be reached sometimes by meditation, in particular in the subjective experience of Samadhi during deep meditation or as the ultimate goal of yoga. Jamieson [18] postulated that the non-reactivity to the contents of awareness in mindfulness practices leads to a heightened level of match between interoceptive predictions and the real felt state of the body[18]. Mindfulness training was reported to induce an increased connection strength of the right insula in all its connections [19]. A larger gyrification within the right antero-dorsal insula was also reported in meditators [20], as well as in increased grey matter volume in the left or right insula, or in the right fronto-insular cortex in a study on a yoga meditation practice[21].

Our findings bring additional support in favor of a major role played by the dorsal anterior insula in the feeling of certainty, accompanied by a feeling of bliss and a sense of timelessness, as observed in ecstatic seizures. The fact that the stimulation of a certain part of the brain is able to induce the complex cognitive state observed in ecstatic auras is extremely interesting. The fact that we have shown an increased functional connectivity of the insula seems to exclude an inhibition of other regions by the abnormal activation of the insula, and favor the above-mentioned hypothesis of a hindrance to the generation of interoceptive prediction errors because of a supra-optimal level of activity inappropriate for this complex cognitive function. We must however recognize that these ecstatic phenomena have only been 
induced in patients with ecstatic epilepsy up to now, and that it is possible that epilepsy changes the excitability and wiring of the regions.

\section{Legends of Table and Figures}

Table 1. Patients' data and stimulation parameters inducing the ecstatic symptom in each patient.

Abbreviations: M, male; F, female; N: normal; FCD: focal cortical dysplasia; OFC: orbitofrontal cortex; ES: epigastric sensation; R: right; L: left; TLE: temporal lobe epilepsy; FLE: frontal lobe epilepsy; ATL: anterior temporal lobectomy; Ant insula: anterior insula.

Figure 1: Anatomical localization of the contacts in the dorsal anterior insula stimulated during ecstatic phenomenon.

A: Localization of the contacts inducing an ecstatic aura in the operculo-insular region in the 3 patients (P1, P2 and P3), in blue. The red dots correspond to other implanted electrodes. Localisation was performed using in-house Gardel software [22]. B: SEEG traces and an example of insular stimulation in patient 3 . Note the absence of after discharges. Intracerebral electrodes are implanted under stereotactic conditions in Talairach's reference frame. Electrodes are identified by letter (A, etc..) and the recordings leads are numbered from 1 to 15 , low numbers corresponding to the deepest structures

Figure 2. Intracerebral EEG functional connectivity changes in the three patients (P1, P2, P3)

A: Functional connectivity graphs are illustrated on a 3D mesh of the MRI with the position of electrodes. Only the significant changes are indicated for the post stimulation period (PoS) relative to the pre stimulation period (PreS). Color scale indicated the $\mathrm{p}$ values of the Zscores. B: for each bipolar channel the degrees are indicated between the two conditions (pre stimulation (PreS) and post stimulation $(\mathrm{PoS}))$. ${ }^{*}$ indicated significant interactions $(* \mathrm{p}<0.05$, $* * \mathrm{p}<0.01$ after Bonferroni corrections). 
Bipolar channels selected for connectivity analysis:

P1: patient 1 (right side): A1-2: Amygdala; B1-2: Anterior hippocampus; I1-2: Insula (middle part); OF3-4: Dorsal insula/Operculum; OR1-2: Orbitofrontal cortex; A9-10: Middle temporal gyrus; OR9-10: Dorsolateral prefrontal cortex.

P2: patient 2 (left side): A'1-2: Amygdala; B'2-3: Hippocampus, IM'1-2: ventral Insula ; OF'1-2: dorsal Insula OR'1-2: Orbitofrontal cortex; B'9-10: Middle temporal gyrus; OR'1011: Dorsolateral prefrontal cortex.

P3: patient 3 (right side): TP1-2: Mesial part of the temporal pole; A1-2: Amygdala; A9-10: Middle temporal gyrus; OR1-2: Orbitofrontal cortex; OR10-11: Dorsolateral prefrontal cortex; PM9-10: Premotor cortex; OF 4-5: dorsal anterior Insula.

Figure 3: Estimation of the leading regions in the network. A: Spheres on each contact represent out-degree values according to the values indicated in the colour scale. B: Mean across time windows of total degree values pooled from the three stimulations (in the three patients). MTL: mesial temporal lobe, OFC: orbito-frontal cortex; TNcx: temporal neocortex. Other: corresponds to different electrodes in the different patients. Highest values are observed for dorsal anterior insula versus mesial temporal lobe $(\mathrm{p}=0.04)$, dorsal anterior insula versus temporal neocortex $(\mathrm{p}=0.04)$; orbitofrontal cortex versus temporal neocortex $(\mathrm{p}=0.005)$, dorsal anterior insula versus orbitofrontal cortex $(\mathrm{p}=0.005)$ and dorsal anterior insula versus other cortex ( $\mathrm{p}=0.04)$ (Wilcoxon test with Bonferoni correction). 


\section{Declaration of Interest:}

Authors have no conflict of interest to disclose

\section{Acknowledgements}

The authors thank Samuel Medina and Bruno Colombet for their help with Anywave and Gardel software.

\section{References}

[1] Rayport SM, Rayport M, Schell CA. Dostoevsky's epilepsy: a new approach to retrospective diagnosis. Epilepsy Behav 2011;22(3):557-70.

[2] Gschwind M, Picard F. Ecstatic Epileptic Seizures: A Glimpse into the Multiple Roles of the Insula. Front Behav Neurosci 2016;10:21.

[3] Picard F, Friston K. Predictions, perception, and a sense of self. Neurology 2014;83(12):1112-8.

[4] Picard F, Craig AD. Ecstatic epileptic seizures: a potential window on the neural basis for human self-awareness. Epilepsy Behav 2009;16(3):539-46.

[5] Picard F. State of belief, subjective certainty and bliss as a product of cortical dysfunction. Cortex 2013;49(9):2494-500.

[6] Picard F, Scavarda D, Bartolomei F. Induction of a sense of bliss by electrical stimulation of the anterior insula. Cortex 2013;49(10):2935-7.

[7] Bressler S. Understanding cognition through large-scale cortical networks. Curr Direct Psych Sci 2002;11(2):58-61.

[8] Badier JM, Dubarry AS, Gavaret M, Chen S, Trebuchon AS, Marquis P, et al. Technical solutions for simultaneous MEG and SEEG recordings: towards routine clinical use. Physiol Meas 2017;38(10):N118-N27.

[9] Colombet B, Woodman M, Badier JM, Benar CG. AnyWave: a cross-platform and modular software for visualizing and processing electrophysiological signals. J Neurosci Methods 2015;242:118-26.

[10] Pijn J, Lopes Da Silva F. Propagation of electrical activity: nonlinear associations and time delays between EEG signals. In: Zschocke, Speckmann, editors. Basic Mechanisms of the EEG, Boston: Birkauser; 1993.

[11] Wendling F, Bartolomei F, Bellanger J, Chauvel P. Interpretation of interdependencies in epileptic signals using a macroscopic physiological model of EEG. Clin Neurophysiol 2001;112:1201-18. 
[12] Courtens S, Colombet B, Trebuchon A, Brovelli A, Bartolomei F, Benar CG. Graph Measures of Node Strength for Characterizing Preictal Synchrony in Partial Epilepsy. Brain Connect 2016;6(7):530-9.

[13] Craig AD. How do you feel--now? The anterior insula and human awareness. Nat Rev Neurosci 2009;10(1):59-70.

[14] Seth AK, Critchley HD. Extending predictive processing to the body: emotion as interoceptive inference. Behav Brain Sci 2013;36(3):227-8.

[15] Mumford D. On the computational architecture of the neocortex. II. The role of cortico-cortical loops. Biol Cybern 1992;66(3):241-51.

[16] Castelhano J, Duarte IC, Ferreira C, Duraes J, Madeira H, Castelo-Branco M. The role of the insula in intuitive expert bug detection in computer code: an fMRI study. Brain Imaging Behav 2018.

[17] Aziz-Zadeh L, Kaplan JT, Iacoboni M. "Aha!": The neural correlates of verbal insight solutions. Hum Brain Mapp 2009;30(3):908-16.

[18] Jamieson G. A unified theory of hypnosis and meditation states: the interoceptive predictive coding approach. In: Lifshitz M, Raz A, editors. Hypnosis and meditation: Towards an integrative science of conscious planes: Oxford University Press; 2015.

[19] Sharp PB, Sutton BP, Paul EJ, Sherepa N, Hillman CH, Cohen NJ, et al. Mindfulness training induces structural connectome changes in insula networks. Sci Rep 2018;8(1):7929.

[20] Luders E, Kurth F, Mayer EA, Toga AW, Narr KL, Gaser C. The unique brain anatomy of meditation practitioners: alterations in cortical gyrification. Front Hum Neurosci 2012;6:34.

[21] Villemure C, Ceko M, Cotton VA, Bushnell MC. Neuroprotective effects of yoga practice: age-, experience-, and frequency-dependent plasticity. Front Hum Neurosci 2015;9:281.

[22] Medina Villalon S, Paz R, Roehri N, Lagarde S, Pizzo F, Colombet B, et al. EpiTools, A software suite for presurgical brain mapping in epilepsy: Intracerebral EEG. J Neurosci Methods 2018;303:7-15. 

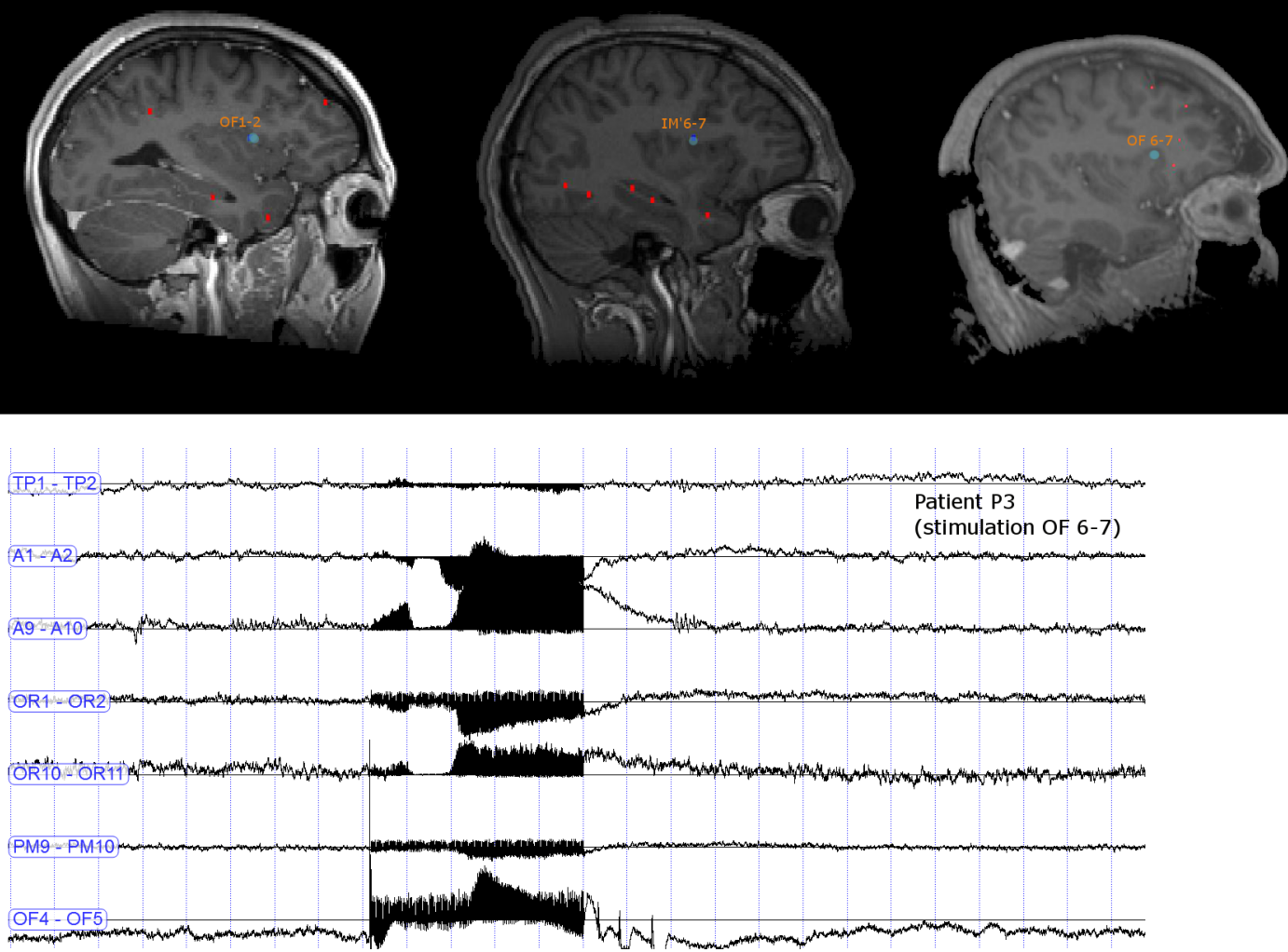

Prestim (PrS)

PostStim (PoS)

Stimulation Artifact 
P1

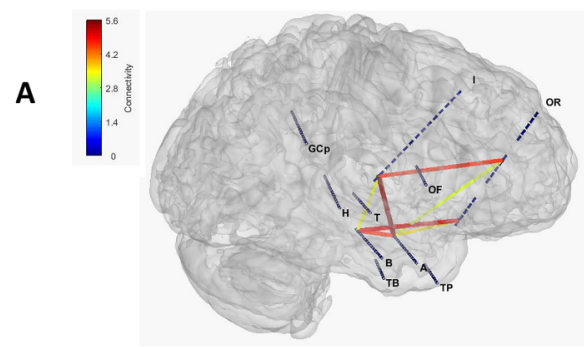

B
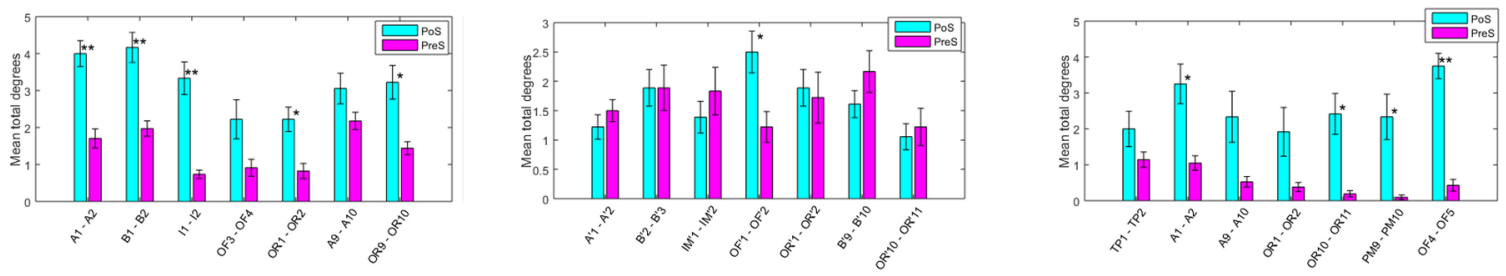
A

P1

P2 Mean out Degrees
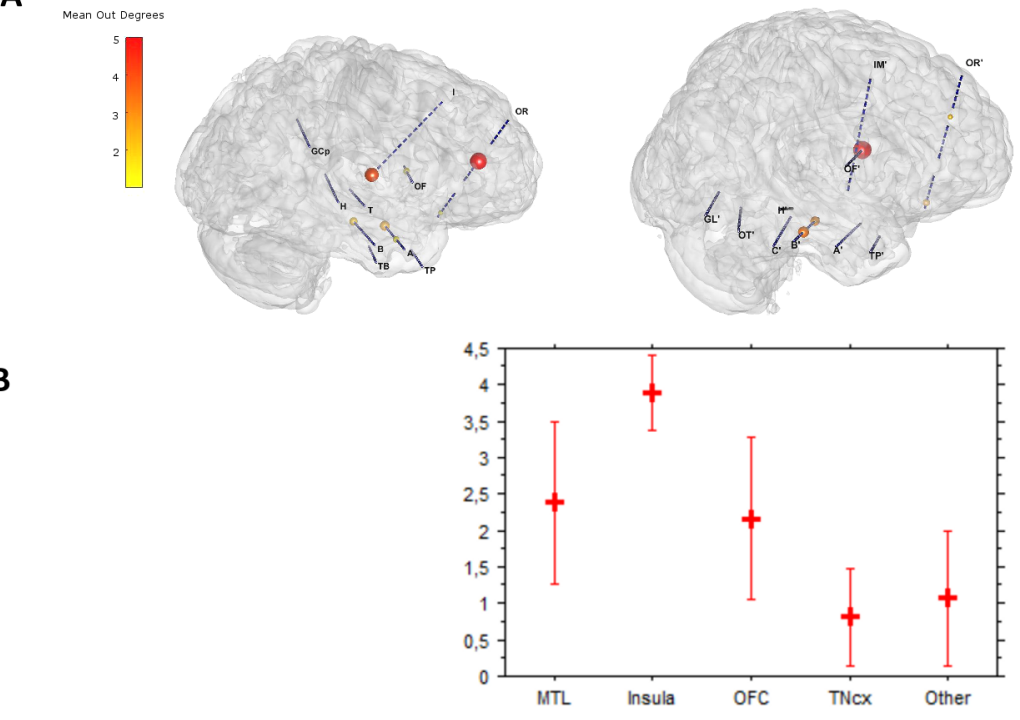

B

P3 


\begin{tabular}{|c|c|c|c|c|c|c|c|c|c|c|}
\hline \multirow[b]{2}{*}{$\begin{array}{l}\text { Patients } \\
\text { (gender) }\end{array}$} & \multirow[b]{2}{*}{ Age (years) } & \multirow[b]{2}{*}{ MRI/etiology } & \multirow{2}{*}{$\begin{array}{l}\text { Spontaneous } \\
\text { sz } \\
\text { subjective } \\
\text { symptoms }\end{array}$} & \multirow[b]{2}{*}{$\begin{array}{l}\text { Type of } \\
\text { epilepsy }\end{array}$} & \multirow[b]{2}{*}{$\begin{array}{l}\text { Surgery (Engel } \\
\text { outcome) }\end{array}$} & \multicolumn{2}{|c|}{$\begin{array}{l}\text { Stimulation inducing } \\
\text { ecstatic symptoms }\end{array}$} & \multirow[b]{2}{*}{ Duration } & \multirow[b]{2}{*}{ Intensity } & \multirow[b]{2}{*}{ Pulse width } \\
\hline & & & & & & Region & Frequency & & & \\
\hline $\mathrm{P} 1(\mathrm{~F})$ & 23 & $\mathrm{~N}$ & Ecstatic, ES & R-TLE & $\begin{array}{l}\text { ATL (outcome } \\
\text { Engel III) }\end{array}$ & Ant Insula & $50 \mathrm{~Hz}$ & $3 \mathrm{sec}$ & $1.6 \mathrm{~mA}$ & $1 \mathrm{~ms}$ \\
\hline $\mathrm{P} 2(\mathrm{M})$ & 18 & FCD & $\begin{array}{l}\text { Ecstatic, ES, } \\
\text { Nausea }\end{array}$ & L-TLE & no & Ant Insula & $50 \mathrm{~Hz}$ & $3 \mathrm{sec}$ & $2.1 \mathrm{~mA}$ & $1 \mathrm{~ms}$ \\
\hline P3 (M) & 36 & OFC lesion & Ecstatic & R-FLE & awaiting & Ant Insula & $50 \mathrm{~Hz}$ & $5 \mathrm{sec}$ & $1.6 \mathrm{~mA}$ & $1 \mathrm{~ms}$ \\
\hline
\end{tabular}

